\title{
Estudio Comparativo de La Variabilidad Del Potencial Toxicogenico de Cepas de Fusarium Graminearum Aislados de Trigo, Cebada Y Maiz Cultivados En Argentina
}

Monica Sepulveda (I), Diana Ramirez Albuquerque (II), Adriana
Kolender (I), Andrea Patriarca (I), Laura Terminiello (II), Virginia Fernandez Pinto (I)

(I) FCEN UBA - Departamento de Quimica Organica (Ciudad Universitaria Buenos Aires Argentina), (II) FCAF UNLP - Universidad Nacional de La Plata (Av 60 y 119 La Plata Buenos Aires Argentina)

\section{Resumo}

Las especies del complejo Fusarium graminearum (FGSC) infectan el trigo, cebada y maíz, causando enfermedades como la fusariosis de la espiga y pudrición de la mazorca. Estas enfermedades reducen el rendimiento del grano y como además el grano infectado suele estar contaminado con micotoxinas constituyen un riesgo para la salud humana y animal. En trabajos previos se identificaron tres perfiles de producción de tricotecenos entre los aislamientos de F. graminearum de trigo (producción de DON y 3acetildeoxinivalenol (3ADON), producción de DON y 15acetildeoxinivalenol (15ADON) y producción de nivalenol (NIV)), siendo la producción de DON y 15 ADON la más frecuente. Existen muy pocos datos para cebada y maiz. El objetivo de este trabajo fue estudiar la variabilidad del potencial toxicogénico de cepas de Fusarium graminearum aislados de trigo, cebada y maíz cultivados en Argentina, para tratar de determinar si el perfil de producción de tricotecenos varía en función del sustrato del cual fueron aislados. Se analizaron muestras de trigo (9), cebada (8), y maíz (7) de la cosecha 2012-2013. Los granos desinfectados superficialmente se colocaron en el medio Dicloran Cloranfenicol Malta Agar y fueron incubados a $25^{\circ} \mathrm{C} 5$ días. Los hongos aislados, una vez 
identificados macro y microscópicamente fueron sembrados en arroz autoclavado e incubados 15 días a 25 y $15^{\circ} \mathrm{C}$ respectivamente. Las toxinas se extrajeron con acetonitrilo/acetato de etilo/agua (50:41:9, v/v) con posterior clean-up en una columna con $0,7 \mathrm{~g}$ de carbón activado, $0,5 \mathrm{~g}$ de alúmina neutra y $0,3 \mathrm{~g}$ de celite y la detección de las mismas se realizó por CG con detector de captura de electrones confirmando por CG-EM. En las muestras de cebada, un 60\% (35/58) de los aislamientos presentaron el perfil DON+ 3-ADON+15-ADON, un 55\% (32/58) el perfil NIV+DON+3ADON+15-ADON y un 9\%(5/58) el perfil NIV+3-ADON. En las muestras de maíz, un 65\% (34/52) presentaron el perfil NIV+ DON+3-ADON+15ADON, un 27\% (14/52) el perfil NIV+15-ADON y un 17\% (9/52) el perfil DON+3-ADON+15-ADON. Para las muestras de trigo, un 56\% (42/75) presentaron el perfil DON+3-ADON+15-ADON y un 35\%(26/75) el perfil NIV+DON+3-ADON+15-ADON. Se observa que el perfil de producción varía según el sustrato de aislamiento ya que el predominante para los aislamientos de maíz fue NIV+DON+3-ADON+15-ADON y para los de trigo y cebada fue DON+3-ADON+15-ADON.

Palavras-Chave: Fusarium graminearum, Tricotecenos, Trigo, Cebada, Maiz Agência de Fomento: 